\title{
Anomaly detection in the concrete arc girder subjected to fatigue test
}

\author{
Dominika Ziaja ${ }^{1, *}$ and Piotr Nazarko ${ }^{1, * *}$ \\ ${ }^{1}$ Rzeszow University of Technology, Powstańców Warszawy 12, 35-959 Rzeszów, Poland
}

\begin{abstract}
A steel reinforced concrete arch of a bridge girder has been subjected to static and fatigue tests. The aim of this study is the application of guided waves in non-destructive diagnostics of civil engineering structures and early damage detection. Two piezoelectric transducers were mounted at a distance of $1 \mathrm{~m}$ to monitor area of the arch keystone. After every 500000 cycles the signals of elastic waves have been measured and the girder visual examination was carried out. It turned out that both the load magnitude and the appearance of cracks have affected the signal changes. The obtained signal database has been used to train artificial neural networks and establish a diagnostic system. The results of the conducted tests have showed good sensitivity of anomaly detection and satisfying accuracy of load identification.
\end{abstract}

\section{Introduction}

The main problems of the bridge construction is a significant reduction in the durability of structures caused by corrosion of steel and concrete. One of the ways to improve the structures safety is to increase the inspections availability and its frequency by the use of structural health monitoring (SHM) systems. They are based on information obtained during non-destructive test. In case of crack detection in concrete structures the impedance-based damage detection method [1, 2], ultrasonic testing [3, 4] or Digital Image Correlation (DIC) [5] are used for this purpose. Also, elastic waves propagation phenomenon is widely applied $[6,7]$ and this approach has been investigated in the presented study.

The fundamentals and possible applications of guided waves is well described in the literature $[8,9]$, especially with respect to simple structures (i.e. beam or plate like structure, made of materials in homogeneous layers) with known boundary conditions. In general, a guided wave excited in the structure, propagates through the structural elements and changes its character (direction, amplitude, etc.) in case of damage appearance and growth (i.e. cracks, delaminations, corrosion, impact damage, yielding zone). This technique has gained considerable popularity in the monitoring of aircraft structures. Nowadays, it is also developed in terms of its applications in the diagnostics of building structures [10-12].

Tracking changes of the propagation character may allow to determine the location and size of damage. However, due to the large number of data, this process requires an adequate signals processing and reasoning algorithm. The dimensionality reduction of measurement data often requires the use of signal compression techniques such as wavelet decomposition,

\footnotetext{
*e-mail: dziaja@prz.edu.pl

**e-mail: pnazarko@prz.edu.pl
} 
Hilbert-Huang transformation or independent component analysis. The approach used in this work is based on a simple transformation which converts the measurement data set into the space of principal components (PCA). It is a kind of lossy compression, but it usually works well in damage detection tasks [13].

An equally important issue is the correct design of the diagnostic system, sensitive to changes associated with the possible damage appearance and its growth. In this case, the inference is usually supported by expert systems, probabilistic methods and artificial intelligence algorithms. In this approach, artificial neural networks were used for this purpose. With their help, an attempt was made to carry out two separate tasks:

- the first was related to the prediction of load force applied to the tested girder - standard neural network (SNN) for regression task was used for this purpose,

- the second one concerned the classification of structure state on the basis of signals registered after each series of fatigue tests - autoasociative neural network (ANN) and novelty index (NI) were adopted in this case.

The application of neural networks allowed the automation of inference process what is a great advantage of the developed diagnostic system due to possible application in the continuous monitoring task.

The second chapter contains a description of the investigated object and the adopted research scope. The third chapter presents the idea of the developed diagnostic system, supplemented with exemplary results of its operation.

\section{Laboratory tests}

The steel reinforced concrete arch of a bridge girder (Fig. 1) has been subjected to static and fatigue tests. Its length and height were 9.95 and $2.29 \mathrm{~m}$ respectively, while the dimensions of the cross-section were 1.00 by $0.28 \mathrm{~m}$. The research programme assumed 2 million cyclic loads. After every 500000 cycles one single static test (up to $120 \mathrm{kN}$ ) and visual examination of the girder was carried out. As a result, the appearing and progressing concrete cracks were marked. In addition to continuous measurements of loads, deformation and stresses, two piezoelectric transducers (Noliac CMAP6, $5 \times 5 \times 2 \mathrm{~mm}$ ) were mounted at a distance of $1 \mathrm{~m}$ on the arch longitudinal axis and around the keystone (at the bottom side, see Fig. 1). They were used in order to excite propagation of elastic wave and to receive the structure response.
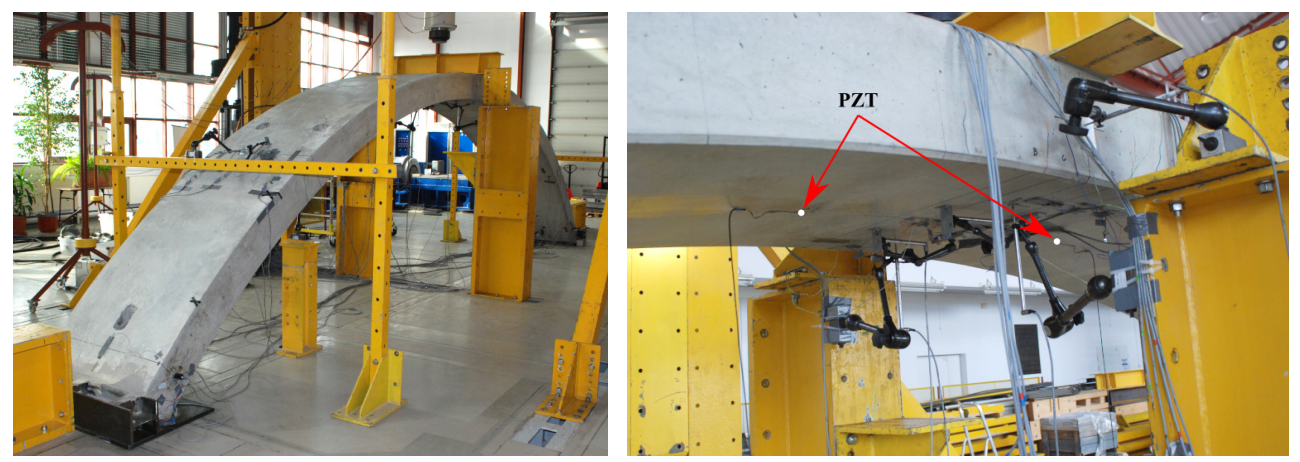

Figure 1. Laboratory model of the concrete bridge girder: general view and location of piezoelectric transducers $(\mathrm{PZT})$ 
During the static tests (an exemplary load history is shown in Fig. 2) the elastic wave signals were stored every $200 \mu \mathrm{s}$. As the excitation pattern 3.5 sine wave, modulated by Hanning window, was used. Its operating frequency was set to $56 \mathrm{kHz}$ and the amplitude to $50 \mathrm{~V}$ (peak-to-peak). This parameters were tuned in order to obtain the highest possible signals to noise ratio.

The received structure response was passing through the charge amplifier (Model 463A, PCB) and then was recorded on a digital oscilloscope (LeCroy WaveRunner) with a sampling frequency of $5 \mathrm{MHz}$. Two examples of registered time signals of elastic wave (under load of $5 \mathrm{kN}$ and $120 \mathrm{kN}$ ) were shown for comparison in Fig. 3. Clear differences between these two signals can be observed. Variance of all collected data in one single static test is shown in Fig. 4. This allows assuming that signal changes are large enough to identify the load or/and deformation magnitude on this basis. It is also worth mentioning (although such a comparison has not been provided in this article) that clear differences in signals were also visible after each of the series of fatigue tests when compared to the same load levels.

(a)

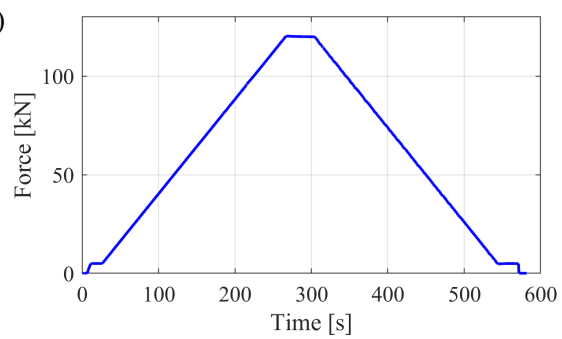

(b)

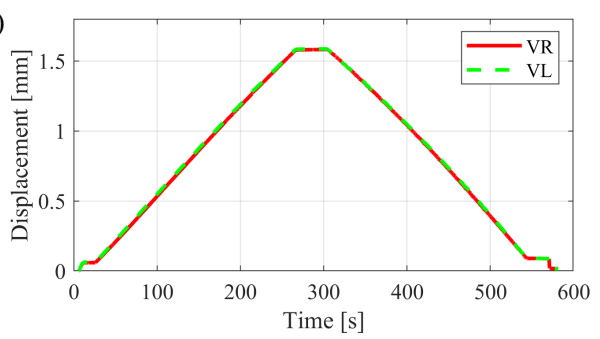

Figure 2. (a) The applied load history and (b) the vertical displacement recorded during static test (VR/VL - right/left side inductive sensor)

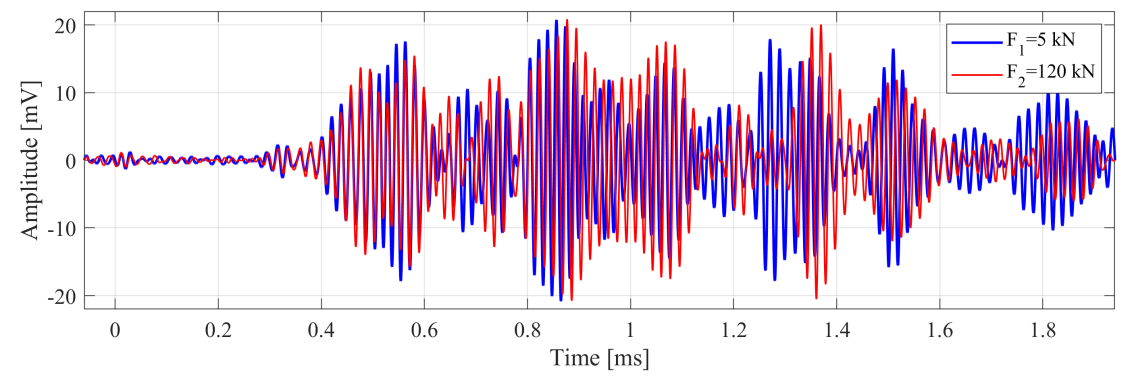

Figure 3. Exemplary time signals of elastic waves recorded in the concrete arch girder subjected to load of 5 and $120 \mathrm{kN}$

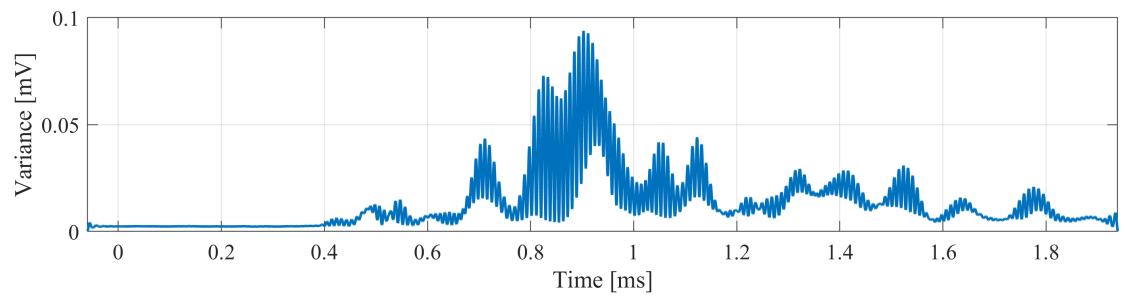

Figure 4. Variance of the elastic waves signals recorded in the concrete arch girder subjected to single static test up to $120 \mathrm{kN}$ 


\section{Training the diagnostic system}

\subsection{Data processing}

On the level of signal processing, the structure responses were filtered using bandpass filter with cut off frequencies set to 32 and $80 \mathrm{kHz}$. Then signal features extraction was performed using PCA, as described in [14]. Input vectors have been created based on the selected number of principal components obtained from the measured time signals. Projections of three first principal components related to elastic wave signals recorded during one single static test and after each series cyclic loads are shown in Fig. 5. Full-length signals were used for this purpose (2 ms). However, the conducted research shows [13] that PCA may be preceded by signals decimation procedure or their analysis may be limited to areas with the highest variability (see Fig. 4). This reduces the size of a coefficient matrix and speeds up the calculations related to PCA.

(a)

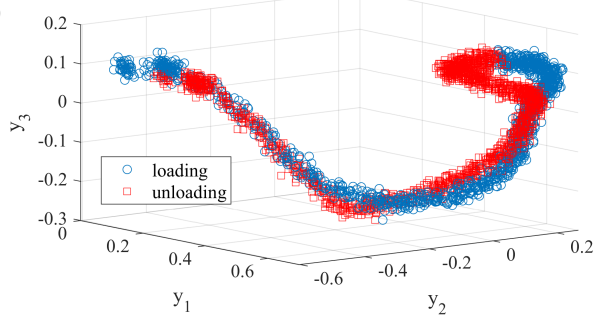

(b)

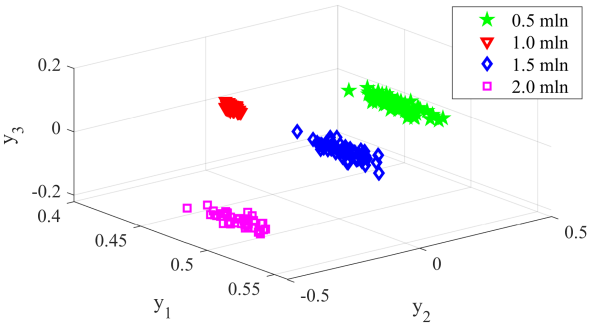

Figure 5. (a) Projection of three first principal components related to elastic wave signals recorded during single static test up to $120 \mathrm{kN}$ and (b) after each series of cyclic loads (comparison for one load level of $5 \mathrm{kN}$ )

\subsection{Load prediction}

Principal components related to single static test performed after 0.5 million cycles (Fig. 5) have defined a first pattern database (2909 patterns were stored). Then, it was divided into learning (1939) and testing sets (970). Every fifth pattern was used for testing (constant distribution). In this task SNN with one hidden layer were used. The size of the input layer was related to the number of considered principal components and was set in this case to 16. The output vector consisted of the load magnitude (see Fig. 2a). Exemplary results of the load prediction using SNN with architecture 16-5-1 are shown in Fig. 6 for both learning and testing patterns. It can be noticed there that very good agreements of the output and target values were achieved. The obtained statistical parameters of the testing error were the following: mean $1.21 \mathrm{kN}$, standard deviation $0.99 \mathrm{kN}$, maximum $6.03 \mathrm{kN}$.

\subsection{Anomaly detection}

This time the patterns database consisted of the principal components related to the load level of $5 \mathrm{kN}$ selected from the static test conducted between every 0.5 million load cycles. It was assumed that each time the structure will be loaded with the same weight, i.e. 20-ton truck standing on or crossing the bridge. In this way 231 patterns were collected, taking into account only first part of the loading stage (see Fig. 2a).

The defined pattern database (Fig. 5b) was then used to train ANNs for the purpose of anomaly detection. An output vector of this neural network is the same as the input vector. Thus, when such a trained ANN is fed with the inputs obtained from a damage state of the 
(a)

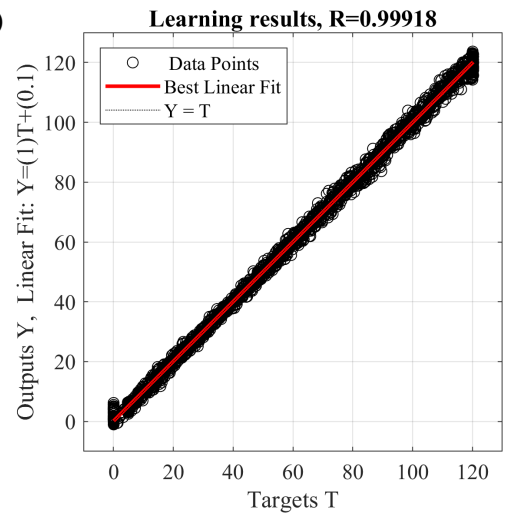

(b)

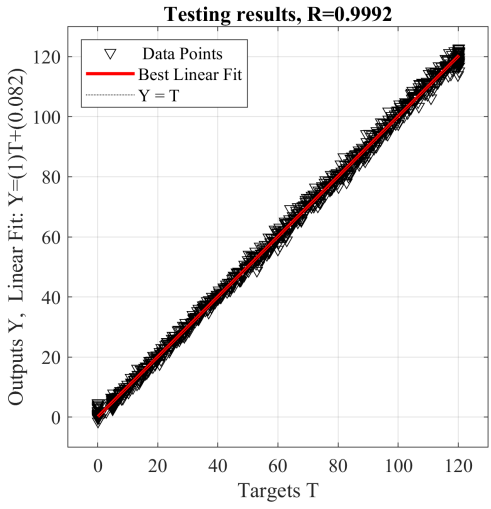

Figure 6. Exemplary results of the load prediction using neural network: a) learning, b) testing

system, the novelty index NI $=\|x-x\|$, which measures the distance between the known input and output of the ANN, will increase $[14,15]$. In this study, feedforward neural networks with one hidden layer were used and they were trained using Levenberg-Marquardt algorithm.

The learning set (76 patterns) was related only to the signals measured after the first series fatigue tests. Every fifth pattern was then selected to the validation set (15 patterns). All other patterns defined the testing set. After ANN training the NI was calculated for every single pattern. Its exemplary values, obtained using ANN's architecture 5-3-5, were shown in Fig. 7.

Introducing certain threshold levels enables not only the anomaly detection but also patterns separation and its classification. The threshold level for the reference set may be calculated as follows: $t=\frac{1}{n} \sum_{i=1}^{n} \mathrm{NI}_{i}+5 \sigma$, where $\sigma$ is a standard deviation calculated for $\mathrm{NI}_{i}$ and $n$ is a number of reference patterns used for training the diagnostic system. Its value was marked in Fig. 7 with a dashed line.

If we hypothetically assume that after a series of 1 million cycles a visual inspection of the structure was carried out and any serious defects were found (i.e. the limit value of the crack width was not exceeded), the recorded signals can be included into the reference set. Exemplary results of anomaly detection obtained in this way were shown in Fig. 8. It can be seen there that also this time the obtained results enable flawless separation of the analysed patterns classes.

\section{Conclusions}

On the basis of experimentally measured elastic wave signals, two diagnostics tasks have been accomplished: 1) prediction of load magnitude, 2) anomaly detection after a series of fatigue

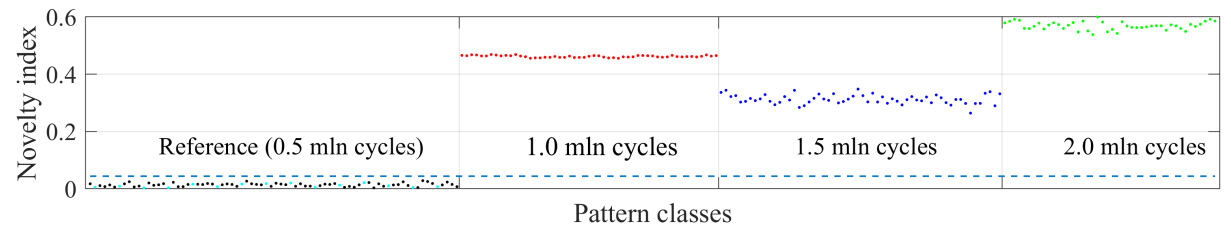

Figure 7. Novelty index calculated on the basis of exemplary results of the ANN training (5-3-5) - the learning set consisted of the patterns related only to the signals measured after the first 0.5 million of cycles 


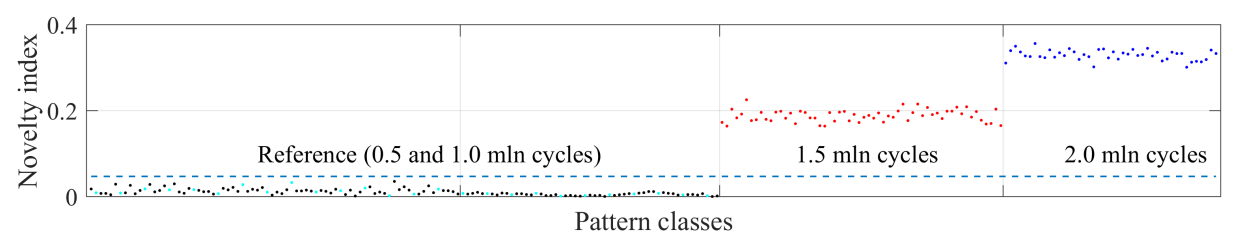

Figure 8. Novelty index calculated on the basis of exemplary results of the ANN training (5-3-5) - the learning set consisted of patterns related to the signals measured after 0.5 and 1.0 million of cycles

tests. The obtained results showed good accuracy and sensitivity of anomaly detection. It was possible thanks to the application of ANN and SNN. The discussed approach enables also the automation of the diagnostic process, which increases both the availability of inspections and the structure's safety.

Unfortunately, at the current stage of research it was not possible to determine how small changes can be detected, because the visual assessment of its condition was carried out periodically after the end of each series of fatigue loads. It can only be concluded that each series of fatigue cycles introduced qualitative changes in the state of the analysed areas, without determining whether they are relevant from the point of view of its safety.

During future tests, it is worth checking if the trained SNN is able to predict load magnitude also in case of the larger number of cycles (e.g. 1 million). In case of anomaly detection the patterns database can be extended with signals measured during unloading stage. An alternative patterns database can be also created when the other load levels are considered, e.g. $120 \mathrm{kN}$. The research plan assumes that in the near future similar tests will be carried out with respect to the concrete arc reinforced using composite fibre bars.

\section{References}

[1] S. Park, S. Ahmad, C.-B. Yun, Y. Roh, Experimental Mechanics, 46 (5), 609-618 (2006)

[2] R. Silva, K. Tsuruta, D. Rabelo, R. Finzi Neto, V. Steffen, EWSHM (2016)

[3] A.A. Shah, Y. Ribakov, Materials \& Design, 30 (9), 3504-3511 (2009)

[4] S. A. Kumar, M. Santhanam, Proc. National Seminar on Non-Destructive Evaluation, Dec. 7-9, 2006, Hyderabad

[5] T. M. Fayyad, J. M. Lees, Procedia Materials Science, 3, 1585-1590 (2014)

[6] G. Zhao, D. Zhang, L. Zhang, B. Wang, Sensors, 18, 4176 (2018)

[7] Y. Lu, J. Li, L. Ye, D. Wang, Constr. Build. Mater., 47, 370-378 (2013) [

[8] T. Stepinski, T. Uhl, W. Staszewski, Advanced Structural Damage Detection. From Theory to Engineering Applications (John Wiley \& Sons, Ltd, 2013)

[9] Z. Su, L. Ye, Identification of Damage Using Lamb Waves. From Fundamentals to Applications (Springer, 2009)

[10] P. Nazarko, MATEC Web of Conferences, 262, 10009 (2019)

[11] M. Rucka, B. Zima, R. Kędra, Archives of Civil Engineering, 60, 493-515 (2014)

[12] J. Li, Y. Lu, R. Guan, W. Qu, Construction and Building Materials, 131, 388-399 (2017)

[13] P. Nazarko, L. Ziemianski, Engineering Failure Analysis, 69, 97-107 (2016)

[14] P. Nazarko, Inverse Problems in Science and Engineering, 21 (6), 945-956 (2013)

[15] M. R. Hernandez-Garcia, M. Sanchez-Silva, Computational Intelligence and Its Applications, Ch. VIII, 158-187 (Idea Group Publishing, 2007) 